\title{
Protective effect of oxymatrine against renal ischemia/reperfusion injury in rats
}

\author{
Ozturk Hulya ${ }^{1}$, Cetinkaya $\mathrm{A}^{2}$, Yilmaz $\mathrm{F}^{3}$, Ozturk $\mathrm{H}^{1}$
}

Abant Izzet Baysal University, Medical School, Pediatric Surgery, Bolu, Turkey. ozturkhayrettin@hotmail.com

\begin{abstract}
BACKGROUND: Renal ischemia/reperfusion (I/R) injury is a common cause of acute kidney injury. The pathologic mechanisms underlying renal I/R injury are complicated, involving reactive oxygen species, necrosis, cell apoptosis, and inflammation, but the exact mechanisms remain unclear.

OBJECTIVES: This study aimed to investigate the effect of oxymatrine (OMT) on renal I/R injury and its underlying mechanism.

METHODS: Thirty male Sprague-Dawley rats were randomly allocated to three groups $(n=10)$ : the sham-control group, the renal I/R-untreated (I/R-untreated) group, and the I/R-OMT group. Renal I/R injury were induced by clamping the left renal artery for $45 \mathrm{~min}$ followed by $24 \mathrm{~h}$ of reperfusion. At $10 \mathrm{~min}$ before reperfusion, the rats in the I/R-OMT-treated group rats received an intravenous injection of $40 \mathrm{mg} / \mathrm{kg}$ OMT. Renal function and histological changes were compared and the relevant parameters of oxidative stress and inflammation were detected. RESULTS: Oxymatrine pretreatment significantly decreased the level of renal dysfunction, attenuated the renal histological changes, the levels of reactive oxygen species production in renal tissue upon I/R. Additionally, OMT pretreatment could further activate the serum antioxidant enzyme activities.

CONCLUSION: The beneficial effects of OMT were likely mediated by the inhibition of lipid peroxidation and the increase in endogenous antioxidant activity. The results of this study indicate that oxymatrine may represent a potent anti-oxidant drug to protect the kidney against I/R injury (Fig. 5, Ref. 29). Text in PDF www.elis.sk. KEY WORDS: oxymatrine, renal ischemia/reperfusion, oxidative stress, rat.
\end{abstract}

\section{Introduction}

Renal ischemia/reperfusion ( $\mathrm{I} / \mathrm{R})$ injury is a common cause of acute kidney injury that occurs in a variety of clinical entities, such as renal resection, hydronephrosis, kidney transplantation, renal artery angioplasty, accidental or iatrogenic trauma, and shock (1-4). The pathologic mechanisms underlying renal I/R injury are complicated and involve oxidative stress, necrosis, apoptosis, calcium ion overloading, and inflammatory reaction $(5,6)$.

In $\mathrm{I} / \mathrm{R}$, tissues are subjected to the destructive proinflammatory effects of cytokine release and the production of oxygen free radicals by neutrophils (7). Therefore, oxidative stress plays an important role in renal I/R injury $(8,9)$. The production of reactive oxygen species (ROS) in the reperfusion period is considered a key reason for uncontrolled oxidative stress, and the increased

${ }^{1}$ Abant Izzet Baysal University, Medical School, Pediatric Surgery, Bolu, Turkey, ${ }^{2}$ Duzce University, Medical School, Physiology, Duzce, Turkey, and ${ }^{3}$ Abant Izzet Baysal University, Medical School, Pathology, Bolu, Turkey

Address for correspondence: H. Ozturk, Dr, Abant Izzet Baysal University, Medical School, Department of Pediatric Surgery, Bolu, Turkey. Phone: +090.374.2534656-3224

Acknowledgement: This work was supported by "Scientific Research Projects Unit"of Abant Izzet Baysal University. amount of ROS can also drive the inflammatory cascade (6). Additionally, cell apoptosis has been considered one of the most serious consequences of renal $\mathrm{I} / \mathrm{R}$ injury in previous studies, and it determines the outcome of renal damage $(10,11)$. The balance between pro- and anti-apoptotic mediators prevents and regulates cell death (12). Therefore, oxidative stress and apoptosis are extremely important in renal I/R injury, and targeting its processes is the ideal therapeutic approach.

Oxymatrine (OMT), a natural quinolizidine alkaloid, is a major bioactive component extracted from the root of traditional Chinese herbal medicine Sophora japonica (sophora flavescesn Ait) (13). OMT has been proved to have such effects as anti-inflammatory, anti-apoptosis, anti-tumor, anti-viral, anti-hypersensitive, and inhibition of histamine release (14). OMT has also been shown to exert a protective effect on ischemia or I/R damage $(15,16)$.

This experiment aimed to investigate the cytoprotective effects of OMT using a rat model of renal I/R injury and to further identify the underlying mechanisms of OMT.

\section{Materials and methods}

All surgical and experimental procedures were approved by the Institutional Animal Care and Use Committee of Abant Izzet Baysal University (Bolu, Turkey) (Number/ID of the approval (s):2015.08.10.905). The procedures were conducted according 

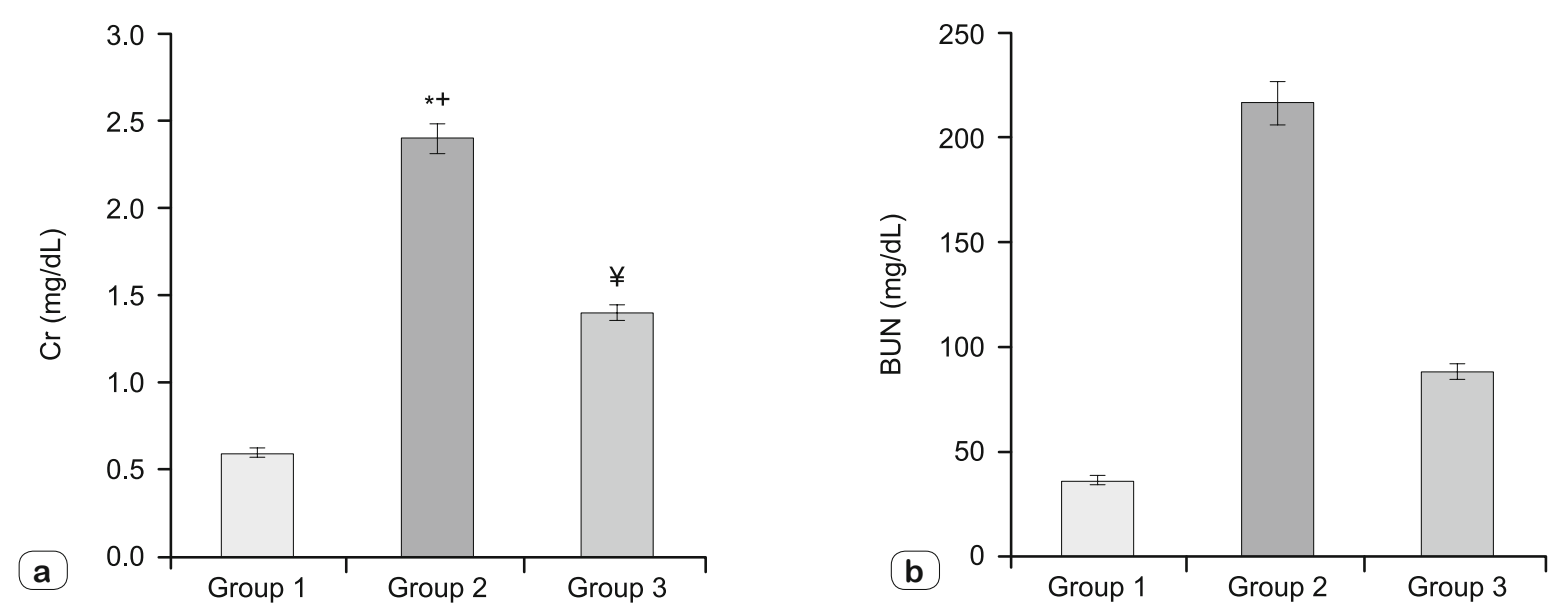

Fig. 1. The effects of oxymatrine (OMT) treatment on alterations of renal function following renal I/R-induced injury. Serum creatinine (a) and urea (a) were measured to assess the reno-protective effect of OMT treatment against renal injury in groups. Data were represented as mean \pm SEM $(\mathbf{n}=10)$. Group 1: sham-operated control; Group 2: I-R/untreated; Group 3: I-R/oxymatrine treated. IR: Ischemia/reperfusion. * $\mathbf{p}<$ 0.0001 (Group 2 vs 1); † p $<0.0001$ (Group 2 vs 3), ¥ p $<0.0001$ (Group 3 vs 1).

a
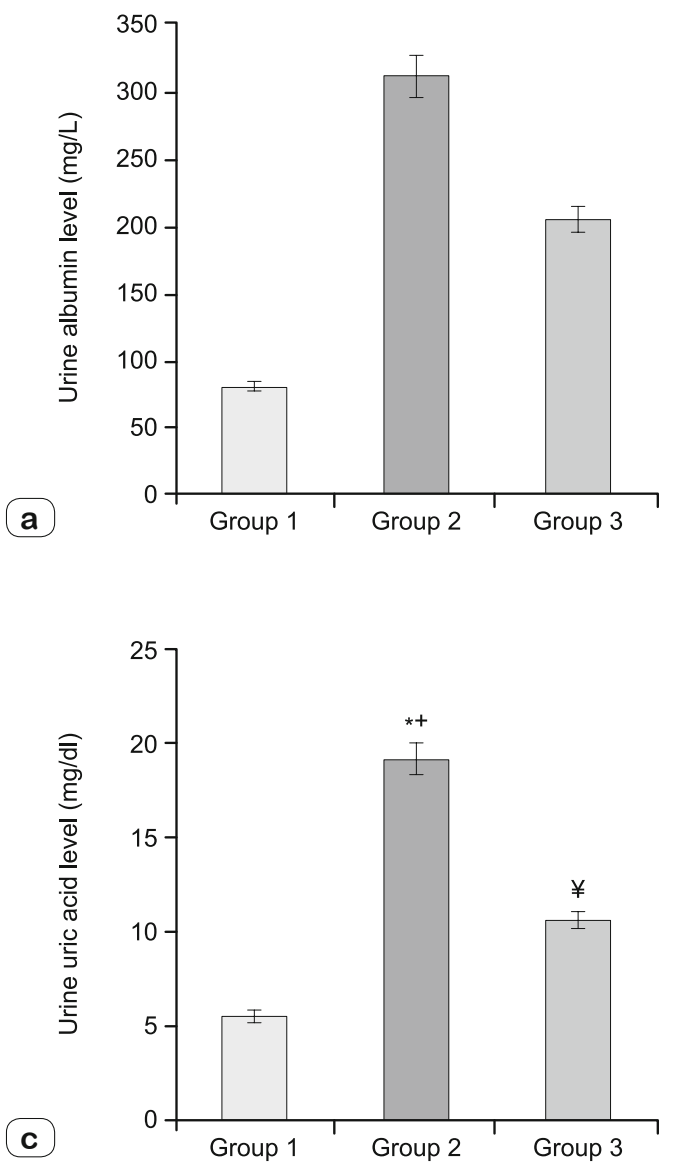

(b)
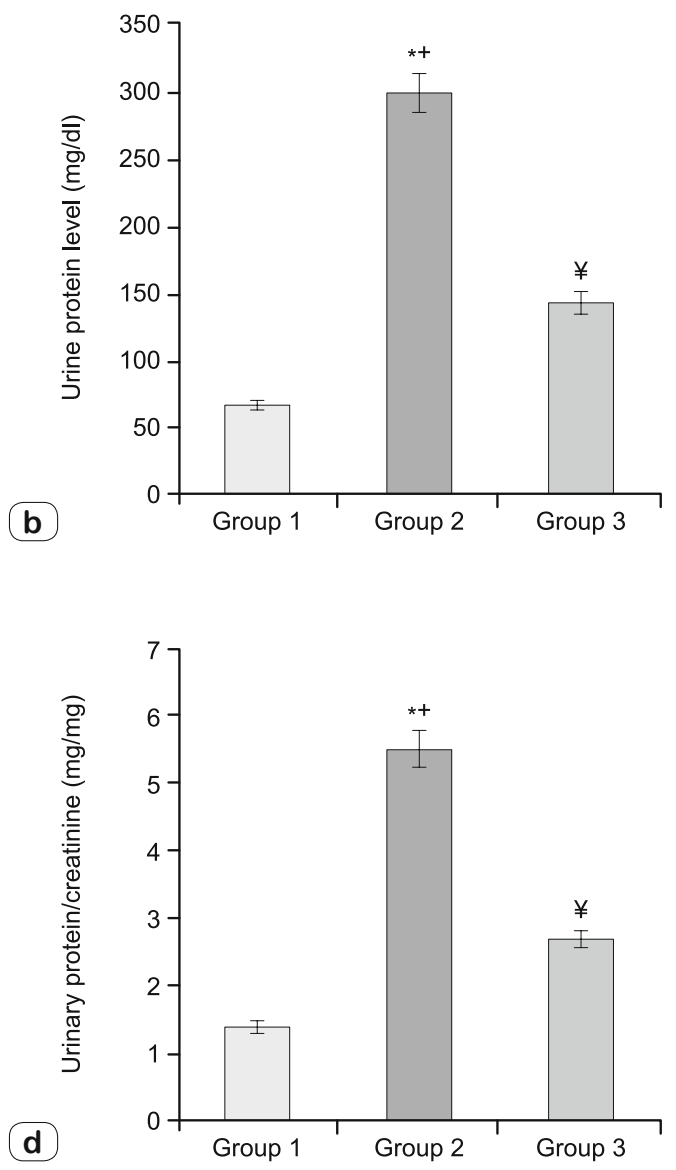

Fig. 2. The levels of urinary albumin (a), protein (b) and uric acid (c) were decreased by OMT treatment in the kidneys exposed to renal ischemia/reperfusion. The ratio of urinary protein/creatinine was significantly increased in the kidneys subjected to IR injury but decreased by OMT treatment (d). Data were represented as mean \pm SEM $(n=10)$. Group 1: sham-operated control; Group 2: I-R/untreated; Group 3: I-R/ oxymatrine treated. IR: Ischemia/reperfusion. ${ }^{*} \mathbf{p}<\mathbf{0 . 0 0 0 1}$ (Group 2 vs 1); †p $<0.0001$ (Group 2 vs 3), ¥ p $<0.0001$ (Group 3 vs 1 ). 
to routine animal care guidelines, and all experimental procedures complied with the Guide for the Care and Use of Laboratory Animals (1996). The animals were all kept in captivity under the same environmental and nutritional conditions. The rats were kept in sawdust-lined cages $(47 \mathrm{~cm}$ x $34 \mathrm{~cm}$ x $18 \mathrm{~cm}$; four animals per cage) in one room at a constant temperature $\left(22 \pm 2{ }^{\circ} \mathrm{C}\right)$, with light from 7:00 $\mathrm{h}$ to $19: 00 \mathrm{~h}$ and water and food ad libitum.

\section{Renal I/R injury}

Briefly, the rats were anesthetized by an intraperitoneal injection of xylazine $(10 \mathrm{mg} / \mathrm{kg})$ and ketamine hydrochloride $(100 \mathrm{mg} /$ $\mathrm{kg}$, Ketalar, Eczacibasi, Turkey) and placed on a homeothermic table to maintain a core body temperature of $37^{\circ} \mathrm{C}$. Following catheterization of the right femoral vein, fluid replacement was performed with $3 \mathrm{~mL} \cdot \mathrm{kg}^{-1} \cdot \mathrm{h}^{-1}$ lactated Ringer's solution using an infusion pump. Then, a midline laparotomy was performed: the right kidney was removed and the left kidney pedicle was shut by an artery clamp for $45 \mathrm{~min}$. After $45 \mathrm{~min}$ of left renal ischemia, occlusion clips were removed and the incision was closed in two layers. The same procedure was performed in the sham operation group without the unilateral clamping process.

The 30 rats were randomly divided into three groups, namely, the sham operation group (Group 1), I/R-untreated group (Group 2), and I/R-OMT-treated group (Group 3), with 10 animals in each group. The sham operation group underwent only the separation of the right renal pedicle but not occlusion. In the I/R-untreated and I/R-OMT-treated groups, the left kidney vessels were clamped for $45 \mathrm{~min}$, followed by $24 \mathrm{~h}$ of reperfusion. At $10 \mathrm{~min}$ before reperfusion, the rats in the I/R-OMT-treated group rats received an intravenous injection of $40 \mathrm{mg} / \mathrm{kg}$ OMT (Sigma-Aldrich-O0891, St. Louis, MO, USA).

We collected 24-h urine samples using metabolic cages. The animals were anesthetized again, blood samples were collected from the abdominal aorta, left kidneys were removed for later analysis, and then the animals were sacrificed.

\section{Preservation of kidneys}

The left kidney was removed under fully maintained anesthesia. After removal, the kidney was fixed in $10 \%$ phosphatebuffered formalin or immediately frozen, and stored at $-80{ }^{\circ} \mathrm{C}$ for different determinations.

\section{Assessment of renal function}

Serum and urinary supernatants were stored at $-80{ }^{\circ} \mathrm{C}$ until laboratory analysis. Samples were thawed before study. Serum BUN, creatinine, and urinary uric acid, creatinine, protein, albumin and the ratio of urinary protein/creatinine analyses were performed with autoanalyzers (Architect c 8000, Abbot Laboratories, USA).

\section{Histopathological evaluation}

The kidney was fixed in $10 \%$ neutral-buffered formalin, paraffin embedded, and sectioned at $4 \mathrm{~mm}$ thickness according to the standard procedure. The sections were deparaffinized and hydrated gradually, and then examined by hematoxylin and eosin staining. Morphologic assessment was performed by an expe- rienced renal pathologist who was unaware of the treatment. A grading scale of $0-4$, as outlined by Jablonski et al (17), was used for the histopathologic assessment of the I/R-induced damage of the proximal tubules.

\section{Measurements of serum antioxidant enzyme activities and lipid peroxidation}

Kidney tissues weighing $100 \mathrm{mg}$ each were placed in labeled glass tubes. Samples were washed with phosphate-buffered saline once and stored at $-80{ }^{\circ} \mathrm{C}$ until the day of biochemical analysis. Samples were thawed before study. Each tissue was homogenized once in $1 \mathrm{ml}$ phosphate-buffered saline. Samples were frozen and thawed twice to facilitate cell membrane disintegration. The homogenate was centrifuged at $4{ }^{\circ} \mathrm{C}$ at $5,000 \mathrm{~g}$ for $5 \mathrm{~min}$, and the supernatant was removed. Catalase (CAT), superoxide dismutase (SOD), and glutathione peroxidase (GSH-PX) activities were measured using commercially available enzyme-linked immunosorbent assay kits according to manufacturer instructions (Cusabio Biotech, Wuhan, PRC). Malondialdehyde (MDA) concentrations were measured using commercially available colorimetric assay kits according to manufacturer instructions (Cayman Chemical Company, Ann Arbor, U.S.A.). BCA protein assay was used for the quantitation of tissue total protein (Thermo Fisher Scientific Inc. Rockford, USA).

\section{Statistical analysis}

All data were analyzed by SPSS 17.0 software. The results are expressed as mean \pm SD. The Kruskal-Wallis test was used for comparison of the groups. In two group comparisons, the Mann-Whitney U test was performed. p values below 0.05 were considered statistically significant.

\section{Results}

The serum BUN and creatinine levels increased in the I/R group compared with those in the sham operation group $(\mathrm{p}<$ 0.0001 and $p<0.0001$, respectively). However, in rats treated with OMT, a reduction in the serum levels of BUN and creatinine was observed unlike in the I/R-untreated group $(\mathrm{p}<0.0001)$ (Fig. $1 \mathrm{a}, \mathrm{b})$. The levels of urinary albumin, protein, and uric acid increased in the kidneys exposed to I/R $(\mathrm{p}<0.0001)$ but decreased in the OMT treatment $(\mathrm{p}<0.0001)$. The ratio of urinary protein/ creatinine also increased by I/R injury $(p<0.0001)$ but decreased by OMT treatment $(\mathrm{p}<0.0001)$ (Fig. $2 \mathrm{a}, \mathrm{b}, \mathrm{c}, \mathrm{d})$.

The value of MDA decreased in the I/R-OMT-treated group ( $p$ $<0.0001$ ) compared with that in the I/R-untreated group. Moreover, the SOD, CAT, and GSH-Px levels increased in the I/R-OMTtreated group compared with those in the I/R-untreated group $(\mathrm{p}<$ $0.0001, \mathrm{p}<0.0001$, and $\mathrm{p}<0.0001$ respectively) (Fig. $3 \mathrm{a}, \mathrm{b}, \mathrm{c}, \mathrm{d}$ ).

The renal injury score (RIS) increased in the experimental groups compared with that in the sham operation $(\mathrm{p}<0.0001$ and $\mathrm{p}<0.0001$, respectively). However, quantitative analysis showed a significantly decreased RIS in the I/R-OMT-treated group compared with that in the I/R-untreated group (Fig. 4). Normative histological changes were found in the sham operation group (Fig. 5 

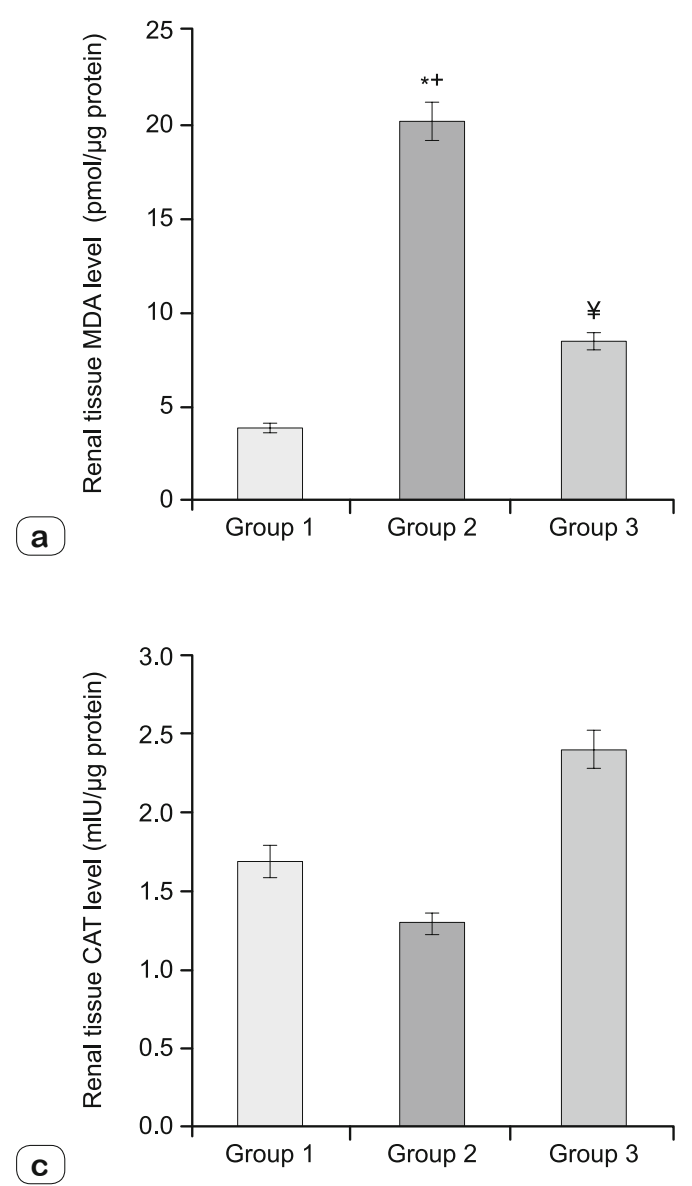
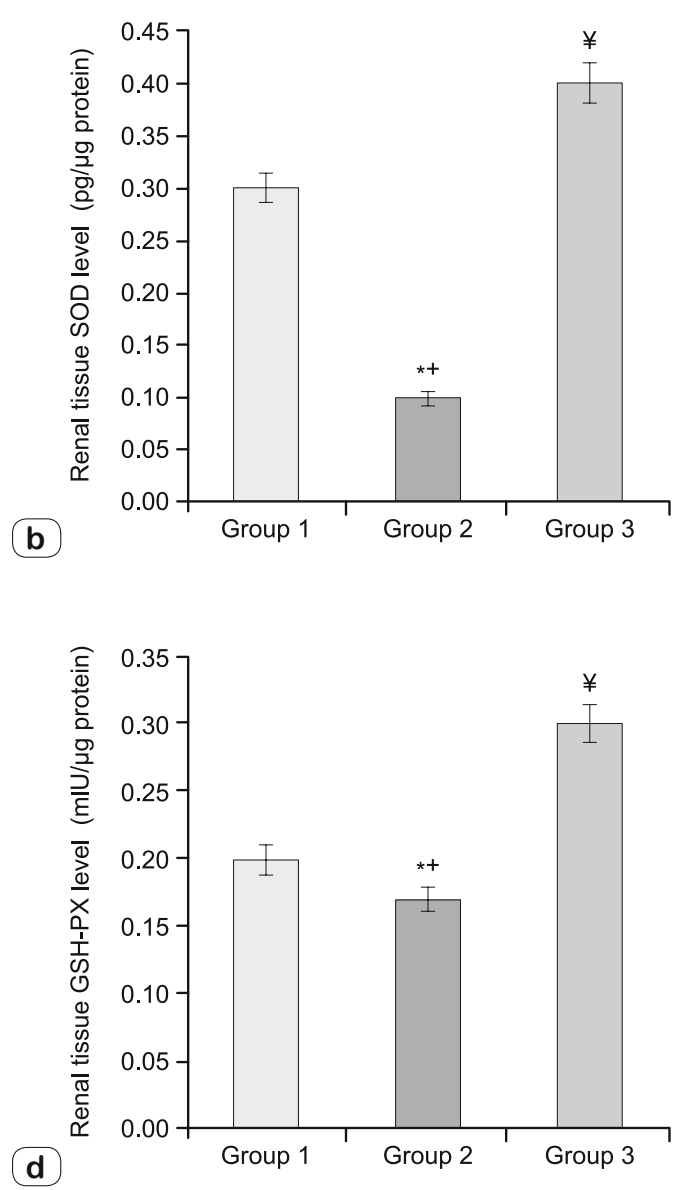

Fig. 3. Malondialdehyde (MDA) was significantly increased in the kidneys subjected to IR injury but decreased by OMT treatment (a). The levels of SOD, CAT and GSH-Px in the renal tissue exposed to renal ischemia/reperfusion were increased by OMT treatment (b, c, d). Data were presented as mean \pm SEM $(n=10)$. Group 1: sham-operated control; Group 2: I-R/untreated; Group 3: I-R/oxymatrine treated. IR: Ischaemia/reperfusion. SOD: superoxide dismutase. CAT: Catalase. GSH-Px: glutathione peroxidase. * $p<0.0001$ (Group 2 vs 1 ); † p $<0.0001$ (Group 2 vs 3), ¥ p $<\mathbf{0 . 0 0 0 1}$ (Group 3 vs 1).

A). Compared with those in the sham operation group, the rats in the I/R-untreated group showed a significant renal histopathologic changes, which included tubular dilation, cloudy swelling and hydropic degeneration in the tubular epithelial cells, vascular congestion and tubular necrosis (Fig. 5 B). In the I/R-OMT-treated group, minimal hydropic changes in the tubular epithelial cells and regeneration of tubular epithelium were observed (Fig. 5 C).

\section{Discussion}

OMT is a natural alkaloid, and its separation and determination have currently matured $(18,19)$. Several studies have shown that OMT has the following pharmacological effects: immune reaction inhibition, anti-viral effect, hepatocyte protection, and anti-hepatic fibrosis effect $(14-16,20,21)$. Additionally, OMT has been reported to be effective because of its anti-apoptotic and anti-inflammatory activities; it can also decrease NF-kappaB expression in myocardial, liver, intestinal, and cerebral I/R injury in animal models (14-16, 22, 23). In this study, the obtained results showed that the renal $\mathrm{I} / \mathrm{R}$ injury induced pathological changes in the renal tissue, such as widespread degeneration of tubular architecture, tubular dilation, tubular cell swelling, cellular vacuolization, inflammatory cell infiltration, severe tubular necrosis, and luminal congestion. The plasma urea and creatinine levels significantly increased in the I/R group compared with those in the control group. The levels of urinary albumin, protein, uric acid, and protein/creatinine were also increased by I/R injury. We found decreased levels of plasma creatinine and BUN and ameliorated pathological changes in the OMT-treated group unlike in the I/Runtreated group. Results from these studies suggested that OMT intravenous injection prior to renal reperfusion significantly attenuated the improvement in the renal dysfunction caused by severe ischemia (45 $\mathrm{min}$ ) and reperfusion in the animals.

The anti-inflammatory and antioxidative effects of OMT, as well as its roles in immunological regulation, have attracted attention in the past few years $(14,22,24)$. OMT has been confirmed to act on nonspecific inflammatory reactions (25). OMT exerts its anti-inflammatory effect independent of the pituitary-adrenal 


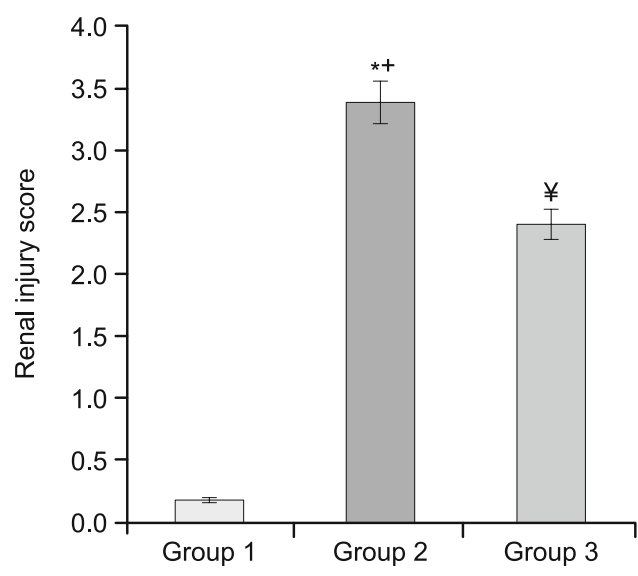

Fig. 4. Jablonski scores for histological appearance of acute tubular necrosis from sham-operated control, I-R/untreated and I-R/oxymatrine treated groups. Renal injury score was significantly increased in the kidneys subjected to IR injury but decreased by OMT treatment. Data were presented as mean \pm SEM. Group 1: sham-operated control; Group 2: I-R/untreated; Group 3: I-R/oxymatrine treated. IR: Ischemia/reperfusion. * p $<0.0001$ (Group 2 vs 1); $\dagger$ p $<0.0001$ (Group 2 vs 3), ¥ $\mathbf{p}<0.0001$ (Group 3 vs 1 ).

system: it acts directly on inflammatory cells (25). OMT is a twoway immunoregulant. That is, it can stimulate the proliferation of lymphocytes at low concentrations but inhibits this proliferation at high concentrations (26). Jiang et al (15) suggested that the protective function of OMT against I/R also depends on its antiinflammatory activity. The release of destructive proinflammatory cytokines during $\mathrm{I} / \mathrm{R}$ is one of the main mechanisms of liver damage, and evidence shows that OMT can inhibit cytokines, such as TNF-a, IL-1, IL-6, and IL-8,14-16, thus supporting the use of oxymatrine to protect the liver against I/R. Zhu et al (27) demonstrated that OMT could significantly induce the expression of IL-10 and downregulate the secretion of TNF-a and IL- 8 from multinuclear leukocytes in a lung $\mathrm{I} / \mathrm{R}$ model in rabbit. Thus, OMT can reduce damage in microvascular endothelial cells, suppress the increasing vascular wall permeability, increase the anti-inflammatory and anti-oxidative capacity of tissues, and increase the stability of cellular membranes.

In the renal ischemia, free radicals generated as a result of lipid peroxidation in the kidney cell membrane cause tissue damage. The best indication of a structural oxidative injury of the cell membrane is the tissue MDA value (28). The biological activity of free radicals is inhibited by antioxidant enzymes SOD and CAT (22). SOD catalyzes the reduction of superoxide anions to $\mathrm{H}_{2} \mathrm{O}_{2}$, which is then converted to $\mathrm{H}_{2} \mathrm{O}$ and $\mathrm{O}_{2}$ by CAT. Moreover, GSH is widely accepted as an important constituent of intracellular protective mechanisms against various noxious stimuli, including oxidative injury (29). In a study on intestinal I/R in rats, Zhao et al (16) showed that OMT significantly attenuated intestinal I/R injury and inhibited cell apoptosis by impeding the production of lipid peroxides and serum levels of TNF- $\alpha$ and by downregulating the expression of phosphorylated p 38 mitogen-activated protein kinase, Fas, and FasL. In another experimental study, Hong et al (22) explored the possible role of OMT against myocardial ischemic damage and several related signaling pathways. They suggested that one of the beneficial effects of OMT was likely mediated by the inhibition of lipid peroxidation and the increase in endogenous antioxidant activity.

As the results of the present study support the notion that depletion of renal GSH, SOD, and CAT and increase in MDA content are some of the major factors causing lipid peroxidation and subsequent tissue damage because of $\mathrm{I} / \mathrm{R}$, the replenishment of renal GSH, SOD, and CAT contents by OMT treatment may be considered as the antioxidant function of OMT. Our recent study also confirms that OMT exerts antioxidant and minimal histopathologic changes and regeneration of tubular epithelium properties in renal tissue by scavenging ROS and increasing the activities of $\mathrm{GSH}$, superoxide dismutase, and catalase.

The current study demonstrated that renal tubular damage and inflammatory cell infiltration caused by renal I/R injury were markedly alleviated in rats following OMT treatment. The beneficial effects of OMT were likely mediated by the inhibition of lipid peroxidation (MDA production) and the increase in endogenous antioxidant activity (CAT, SOD, and GSH-PX). This finding indicates that OMT exhibits renoprotective effects against renal I/R injury by inhibiting oxidative stress.
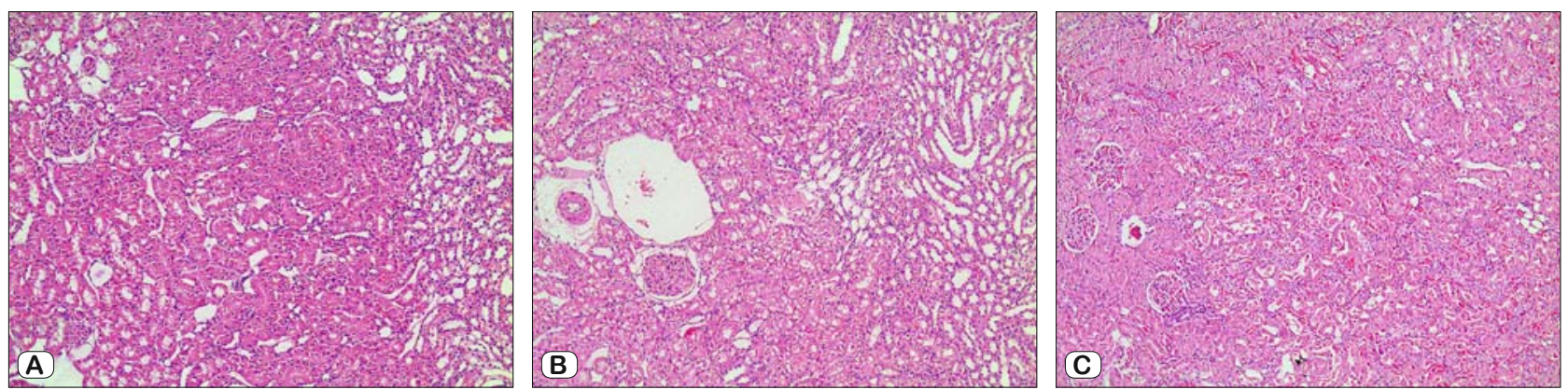

Fig. 5. Representative kidney sections stained with H\&E at the end of 24 hours' reperfusion. A) Sham-operated animals: normal histological characteristic of glomeruli and tubules. B) Rats subjected to renal I-R injury: marked necrosis with tubular dilation, swelling, and luminal congestion. C) Rats subjected to renal I-R injury, pretreated with Oxymatrine: minimal hydropic changes in the tubular epithelial cells and regeneration of tubular epithelium. (H\&E, x100). I-R: Ischaemia/reperfusion. 


\section{References}

1. Sagiroglu T, Torun N, Yagci M, Yalta T, Sagiroglu G, Oguz S. Effects of apelin and leptin on renal functions following renal ischemia/ reperfusion: An experimental study. Exp Ther Med 2012; 3 (5): 908-914.

2. Yun Y, Duan WG, Chen P, Wu HX, Shen ZQ, Qian ZY, Wang DH. Ischemic postconditioning modified renal oxidative stress and lipid peroxidation caused by ischemic reperfusion injury in rats. Transplant Proc 2009; 41 (9): 3597-3602.

3. Snoeijs MG, Vink H, Voesten N, Christiaans MH, Daemen JW, Peppelenbosch AG et al. Acute ischemic injury to the renal microvasculature in human kidney transplantation. Am J Physiol Renal Physiol 2010; 299 (5): F1134-1140.

4. Wang Y, Seto SW, Golledge J. Therapeutic effects of renal denervation on renal failure. Curr Neurovasc Res 2013; 10 (2): 172-184.

5. Zhang J, Zou YR, Zhong X, Deng HD, Pu L, Peng K, Wang L. Erythropoietin pretreatment ameliorates renal ischaemia/reperfusion injury by activating PI3K/Akt signalling. Nephrology (Carlton) 2015; 20 (4): 266-272.

6. Wang L, Liu X, Chen H, Chen Z, Weng X, Qiu T, Liu L. Effect of picroside II on apoptosis induced by renal ischemia/reperfusion injury in rats. Exp Ther Med 2015; 9 (3): 817-822.

7. Barinaga M. Forging a path to cell death. Science 1996; 273 (5276): 735-737.

8. Eltzschig HK, Eckle T. Ischemia and reperfusion - from mechanism to translation. Nat Med 2011; 17 (11): 1391-1401.

9. Brooks C, Wei Q, Cho SG, Dong Z. Regulation of mitochondrial dynamics in acute kidney injury in cell culture and rodent models. J Clin Invest 2009; 119 (5): 1275-1285.

10. Zhang ZX, Shek K, Wang S, Huang X, Lau A, Yin Z et al. Osteopontin expressed in tubular epithelial cells regulates NK cell-mediated kidney ischemia reperfusion injury. J Immunol 2010; 185 (2): 967-973.

11. Lin M, Li L, Li L, Pokhrel G, Qi G, Rong R, Zhu T. The protective effect of baicalin against renal ischemia/reperfusion injury through inhibition of inflammation and apoptosis. BMC Complement Altern Med 2014; 14: 19 .

12. Selzner M, Rüdiger HA, Selzner N, Thomas DW, Sindram D, Clavien PA. Transgenic mice overexpressing human Bcl-2 are resistant to hepatic ischemia and reperfusion. J Hepatol 2002; 36 (2): 218-225.

13. Zhang K, Li YJ, Yang Q, Gerile O, Yang L, Li XB et al. Neuroprotective effects of oxymatrine against excitotoxicity partially through down-regulation of NR2B-containing NMDA receptors. Phytomedicine 2013; 20 (3-4): 343-350.

14. Liu Y, Zhang XJ, Yang CH, Fan HG. Oxymatrine protects rat brains against permanent focal ischemia and downregulates NF-kappaB expression. Brain Res 2009; 1268: 174-180.
15. Jiang H, Meng F, Li J, Sun X. Anti-apoptosis effects of oxymatrine protect the liver from warm ischemia reperfusion injury in rats. World $\mathrm{J}$ Surg 2005; 29 (11): 1397-1401.

16. Zhao J, Yu S, Tong L, Zhang F, Jiang X, Pan S et al. Oxymatrine attenuates intestinal ischemia/reperfusion injury in rats. Surg Today 2008; 38 (10): 931-937.

17. Jablonski P, Howden BO, Rae DA, Birrell CS, Marshall VC, Tange J. An experimental model for assessment of renal recovery from warm ischemia. Transplantation 1983; 35 (3): 198-204.

18. Ku YR, Chang LY, Lin J, Ho LK. Determination of matrine and oxymatrine in Sophora subprostata by CE. J Pharm Biomed Anal 2002; 28 (5): 1005-1010.

19. Song JZ, Xu HX, Tian SJ, But PP. Determination of quinolizidine alkaloids in traditional Chinese herbal drugs by nonaqueous capillary electrophoresis. J Chromatogr A 1999; 857 (1-2): 303-311.

20. Coon JT, Ernst E. Complementary and alternative therapies in the treatment of chronic hepatitis C: a systematic review. J Hepatol 2004; 40 (3): 491-500.

21. Ma SC, Du J, But PP. Antiviral Chinese medicinal herbs against respiratory syncytial virus. J Ethnopharmacol 2002; 79 (2): 205-211.

22. Hong-Li S, Lei L, Lei S, Dan Z, De-Li D, Guo-Fen Q et al. Cardioprotective effects and underlying mechanisms of oxymatrine against ischemic myocardial injuries of rats. Phytother Res 2008; 22 (7): 985-989.

23. Cui L, Zhang X, Yang R, Wang L, Liu L, Li M, Du W. Neuroprotection and underlying mechanisms of oxymatrine in cerebral ischemia of rats. Neurol Res 2011; 33(3): 319-24.

24. Fan C, Zwacka RM, Engelhard JF. Therapeutic approaches for ischemia/reperfusion injury in the liver. J Mol Med 1999; 77 (8): 577-592.

25. Liu F, Liu J, Chen X, Lv WW, Zheng JZ. Effect of oxymatrine on inflammation and its mechanism. Journal of Jilin University Medicine Edition 2005; 31: 728-730.

26. Fan H, Li L, Zhang X, Liu Y, Yang C, Yang Y, Yin J. Oxymatrine downregulates TLR4, TLR2, MyD88, and NF-kappaB and protects rat brains against focal ischemia. Mediators Inflamm 2009; 2009: 704-706.

27. Zhu B, Yang JR, Chen SF, Jiang YQ. The Attenuation of Lung Ischemia Reperfusion Injury by Oxymatrine. Cell Biochem Biophys 2014; 70 (1): 333-336.

28. Akdere H, Tastekin E, Mericliler M, Burgazli KM. The protective effects of Ginkgo biloba EGb761 extract against renal ischemia/reperfusion injury in rats. Eur Rev Med Pharmacol Sci 2014; 18 (19): 2936-2941.

29. Ross D. Glutathione, free radicals and chemotherapeutic agents. Mechanismsof free-radical induced toxicity and glutathione-dependent protection. Pharmacol Ther 1988; 37 (2): 231-249.

Received December 12, 2016. Accepted December 23, 2016. 